\title{
LES ÉCRITS D'ARTISTE : ENTRE RÉFLEXION CRITIQUE ET CRÉATION ARTISTIQUE
}

\section{ARTIST'S WRITINGS: BETWEEN CRITICAL REFLECTION AND ARTISTIC CREATION}

Lorena Garcia Cely Université de Paris 8 École d'Esthétique, Sciences et Technologies des Arts Laboratoire Arts des Images et Art Contemporain, Paris, France

Résumé : Un trait distinctif de l'art des années cinquante et soixante est la production significative d'écrits d'artiste. Ils sont pour la plupart publiés dans des revues d'art, notamment, à Paris et à New York. Dans son ensemble, ces textes ont révélé le besoin des artistes d'évaluer de manière critique leur travail. Cet article se centre sur ceux dans lesquels, en plus de cela, existe une dimension esthétique qui fait aussi partie de l'espace discursif. L'objectif est d'explorer ce type de discursivité. Ceci afin de montrer que l'écriture artistique, se trouve à l'origine d'une nouvelle forme discursive, où la compréhension critique présuppose également l'interprétation des qualités esthétiques du texte.

Mots-clés : Écrits d'artiste ; revue d'art ; discours ; critique d'art ; esthétique.

Abstract: A distinctive feature of the art of the fifties and sixties is the significant production of writings authored by the artists themselves. These were mostly published in art magazines, particularly in Paris and New York. As a whole, these texts showed the artist's need for a critical evaluation of her or his work. The present article focuses on those in which, in addition to this, an aesthetic dimension finds its way through the discourse. Our aim is to explore this type of discourse shape in order to show that the artistic writing is at the origin of a new discourse form, in which the critical understanding also implies the appreciation of the aesthetic qualities of the text.

Keywords: Artist's writings; Art magazines; speech; art critic; aesthetics.

\section{Les écrits d'artiste dans les revues d'art à Paris et New-York}

Nombreux sont les artistes qui dans le tournant de la deuxième moitié du XXème siècle ont mené un travail d'écriture, inscrit au cœur de 
leurs pratiques artistiques, ou bien, parallèle à leurs activités créatives. Devenant un outil important pour le positionnement de leur pensée au sein du monde de l'art, leurs écrits couvrent diverses catégories discursives et leur publication s'étend à divers supports. D'après une étude proposée par Olivier Mignon, parmi les nombreux textes diffusés, entre tracts, livres, catalogues d'exposition et périodiques, ce sont ces derniers qui leur ont octroyé un espace considérable (MIGNON (éd.), 2010, p. 5). Ce phénomène est corrélé au fait qu'à partir de 1945, des nombreuses revues relevant du domaine artistique sont née un peu partout dans le monde, notamment à Paris et New-York (ALLEN, 2011, p. 227).

Des deux côtés de l'Atlantique, plusieurs artistes ont donc fait des contributions au monde des périodiques. Quelques-uns s'engagent dans des projets de grande envergure afin de défendre leurs postures artistiques. Pour cela, ils fondent ou ils font partie du comité éditorial des revues d'art. Leur travail est autant en lien avec le propos de la revue, qu'en fonction de leurs propres démarches créatives. Les artistes qui participent aux publications et qui ont une grande responsabilité éditoriale, le font dans la plupart des cas, pour exprimer et transmettre leur conception de l'art. Ainsi, leur travail ne se limite pas à des tâches administratives. Ils sont présents pour faire avancer les objectifs des revues car eux-mêmes ont besoin de partager leurs idées. Quelques autres, limitent leur participation à un travail d'écriture occasionnel, mais constant. Faisant des apports réguliers, ils ont un espace de parole dans chaque publication. Et finalement pour d'autres artistes, l'écriture est un geste exceptionnel.

De même, ces écrits couvrent diverses catégories littéraires ainsi que supports matériels variés. Plusieurs font appel aux compositions libres, comme le rappelle le roman Manhattan suicide addict de l'artiste Yayoi KUSAMA (2005) ; certains suivent les formalités académiques de l'essai et de la dissertation philosophique tel que le Postface : un journal critique de l'avant-garde de Dick HIGGINS (1964); d'autres font partie du registre journalistique comme les articles d'Arthur SECUNDA chez Artforum (1962, 1963) ; une anthologie nourrie également par les livres d'artiste et les œuvres conceptuelles où les textes sont autant de l'art que des réflexions théoriques (MCEGLIN-DELCROIX, 1997).

Face à une telle dissemblance textuelle, leur contenu peut être lu de la même façon qu'un quelconque écrit, alors que peut-être l'artiste éprouve le besoin de voir leurs écrits perçus comme une extension de sa pratique? Comment peut-on concilier le problème entre la forme et le contenu que 
posent ces écrits lorsqu'il s'agit de saisir leur sens?

\section{Les artistes et la critique de l'art}

Les écrits d'artiste touchent différents champs discursifs. Étant une sorte de laboratoire d'exploration de genres, leur lien commun est de promulguer des jugements sur l'art. Ils s'inscrivent donc dans le domaine de la critique de l'art.

Jean-Pierre Cometti, Jacques Morizot et Roger Pouivet, définissent celle-ci comme une action dans laquelle s'élabore un discours axé principalement sur la singularité des créations artistiques. D'après eux, le critique d'art construit un jugement issu du rapport entre quatre opérations, lesquelles ne peuvent pas exister indépendamment : celle « de la description (puisque le critique doit rendre compte d'une rencontre sensible et particulière avec une œuvre particulière), de l'évaluation (puisque le critique juge ou apprécie la qualité, la réussite ou l'échec de l'œuvre), de l'interprétation (puisque le critique dégage un contenu ou un sens), de l'expression (puisque le critique exprime ses choix, ses conceptions, ses goûts, ses sentiments " (COMETTI, MORIZOT, POUIVET, 2000, p. 157). Selon cette définition, tant qu'un argument comporte la description d'une œuvre d'art et son évaluation, ce qui supposerait son interprétation, on est face à un jugement critique. L'écriture est la manière dont l'artiste, décrit, évalue interprète et porte la défense de ses propres pratiques et celles d'autrui.

Défendre quelque chose, c'est reconnaître qu'il existe un pouvoir légitimateur auquel on est soumis. Les artistes écrivent, en effet, en cherchant à gagner le sérieux accordé à la parole des spécialistes du monde de l'art afin de s'emparer du pouvoir de décider la valeur de leurs œuvres. Ainsi, ces textes interrogent le fait que certains jugements soient plus légitimes que d'autres, non pas par rapport aux arguments mêmes, mais par rapport à la personne qui les dispense. Selon Laurence CORBEL, à partir de la deuxième moitié $\mathrm{du} \mathrm{XX}^{\text {ème }}$ siècle, il en est ainsi car les artistes contestent de plus en plus « la mainmise de toute autorité étrangère sur leurs œuvres» (2012, p. 47). Dans ce sens, les artistes remettent en question les aptitudes discursives ainsi que le positionnement institutionnel de ceux qui, avec ses formes, imposent des vérités artistiques aux artistes.

Cela ouvre la question de la dimension objective des affirmations portées par les artistes. S’il n'existe pas une séparation entre celui qui juge 
et ce dont est jugé, l'écriture devient-elle une réflexion subjective éloignée de toute forme de vérité ? Si un critique d'art ou un auteur académique ont également une posture à défendre, concernant les artistes, la question serait plutôt à déplacer vers le degré de subjectivité qu'accompagnent les jugements ? Si l'artiste est attaché directement par la critique qu'il fait de son œuvre, à quel type de critique cela donnerait lieu?

\section{Trois types de textes, les mêmes enjeux discursifs}

Un premier exemple de la critique élaborée par les artistes, est celle où l'expérience de vie est mentionnée pour donner du poids à l'argumentation. En effet, certains mettent en avant leur vie afin de souligner son évolution et ainsi justifier la valeur de leurs œuvres. Dans cette forme d'écrit, les artistes placent leur pratique dans une ligne temporale. Les critères à partir desquels ils analysent leurs travaux, sont donc donnés par leur propre perception sur la progression de sa pratique. La liberté offerte par ce cadre d'analyse, permet à l'artiste de faire intervenir dans l'écrit, des passages d'ordre littéraire, afin d'illustrer ses propos.

Dans le numéro de décembre 1965 de la revue Artforum se trouve l'écrit de Dan Flavin ... "in daylight or cool white ». An Autobiographical Sketch. En évoquant l'ensemble de son œuvre, l'auteur développe une dimension descriptive, analytique, évaluative mais également expressive. C'est un récit personnel où, de manière détaillée, l'artiste présente différents moments de sa vie et sa carrière. Éloignée des postures académiques, celui s'approche plus parfois du travail poétique: «I knew that the actual space of a room could be broken down and played with by planting illusions of real light (electric light) at crucial juncture in the room's composition " (FLAVIN, 1965, p. 22). Dans plusieurs passages de l'article, l'auteur expose une réflexion sur son art, tout en délivrant une sorte de travail littéraire. Le fil discursif conduit ainsi le lecteur vers une double lecture, l'une critique, l'autre littéraire.

Pareillement, dans le numéro de janvier 1966 parait Extracts from the Studio Notes de l'artiste Claes Oldenburg. De la même manière que Flavin, Oldenburg évoque différents moments de sa vie, lesquels ont, selon lui, déterminé sa pratique. Par le biais de son texte, il présente une sorte de logique qu'il considère inscrite dans son travail et laquelle est en lien avec son expérience de vie. Et, comme dans le cas de Flavin, son écriture comporte des aspects littéraires: «My art gives the (deliberate) impressions 
of being concerned with the outside world... but in fact it is simply the personal elaboration of imaginary forms... of a limited number... in the guise of occasional appearances..." (OLDENBURG, 1966, p. 33).

Dans les cas de ces deux artistes, il s'agit d'élever à la catégorie critique, leurs impressions sur leurs vies en lien avec leurs pratiques. Les deux écrits sont dans une démarche tant descriptive qu'évaluative. S'attardant sur les évidences données par l'expérience, ces textes permettent de mieux connaître ce à quoi ils tiennent en tant qu'artistes; ainsi que la valeur qu'ils estiment, comporte leurs œuvres. La critique est a priori bienveillante vis-à-vis d'une sorte de progression conceptuelle et technique. Cette caractéristique, cependant, ne conduit pas nécessairement à une manière d'écriture critique d'ordre institutionnel. Or, étant donné le rapport à la subjectivité de chaque texte, soit on assume que le travail critique de ces écrits n'est pas légitime ; soit on cherche des nouveaux référents pour juger leur pertinence critique.

Un deuxième exemple de cette forme critique développée par les artistes, est celle où sans évoquer des expériences personnelles, ils parlent de l'ensemble de leur travail de la façon la plus objective possible. Une revue mettant en évidence ce type de texte est Leonardo.

Fondée en 1967 par Frank Joseph Malina, cette revue est conçue comme un espace d'échange pour les artistes désireux de partager des nouveautés technologiques dans l'art, avec une rigueur scientifique. ${ }^{1}$ Les écrits, pour la plupart élaborés par des artistes, comptent avec de résumés expliquant les propos de l'auteur, des argumentaires élaborés à la façon de textes scientifiques et de citations ayant un format académique. Ainsi, même si Leonardo n'est pas une revue avec un comité scientifique évaluant la recherche effectuée par les participants, elle a permis de tisser de liens entre les artistes. Le but de Malina était, en effet, de construire un support agissant comme un réseau scientifique que serait le plus large possible.

Deux exemples d'articles témoignent de ces propos. D'abord, l'artiste Georges Noël dans Reliefs explique en quoi consiste son travail. Celui est constitué par des tableaux sculptés ou, comme lui même les nomme, des sculptures de peintre (NOËL, 1968, p. 77) ; des œuvres qu'il estime proches de l'architecture. C'est un texte descriptif, accompagnés de nombreux commentaires sans fondement scientifique. "En parlant de peinture et de sculpture, on entend souvent s'introduire la distinction entre deux et trois dimensions. Cependant, il ne faut pas se confondre. La peinture n'est pas

\footnotetext{
${ }^{1}$ Toutes les informations publiés dans cette partie ont été tirées du site officiel de la revue : https://www.leonardo.info/history. Site internet consulté le 4 novembre 2018.
} 
3-1=2 dimensions, pas plus que la sculpture serait $2+1=3$ dimensions. La troisième dimension telle que je la conçois est présente dans les deux cas. Elle est celle de la lumière " (NOËL, 1968, p. 77). Noël considère que la profondeur n'apporte pas la troisième dimension, mais la lumière. C'est un affirmation qui fonde les explications sur son travail : « ce que j'ai voulu c'est que le tableau en tant que surface à deux dimensions projetant sa propre lumière devienne escalier qui descend dans l'espace afin d'accrocher la lumière ambiante " (NOËL, 1968, p. 77). Formellement, telle que la revue le souhaite, le texte montre la pensée de l'artiste, ses travaux et ses projets en cours. Conceptuellement, celui est un apport personnel à mi chemin entre la critique et des affirmations plutôt littéraires. Alors, bien que le texte garde une cohérence argumentative, l'on peut se poser la question de si cela suffit pour qu'il soit apprécié scientifiquement; c'est-à-dire qu'il soit estimé par rapport à la qualité de ses arguments du point de vue de la vérité.

En outre, l'artiste-scientifique Frank J. Malina, participe à la publication avec le texte Kinetic painting: The Lumidyne system. C'est une dissertation expliquant le système de création qu'il a inventé et nommé Lumidyne :

I developed in 1956 the main composition is painted on a fixed, transparent plate (the Stator), and a design is painted on a rotating transparent disc (the Rotor). Light from incandescent bulbs or florescent tubes is transmitted directly through the Rotor and then the Stator on to a translucent plate (the Diffusor) to produce a picture combining light, color and movement. I have called this the Lumidyne system. (MALINA, 1968, p. 33)

Expliquant chaque partie qui intègre son système avec des images à l'appui, l'évolution de la machine est dévoilé au lecteur pas à pas. De même, l'artiste se consacre à l'éclaircissement des notions nécessaires à la compréhension des peintures produites par la machine : mouvement, couleur, changement, considérations esthétiques. Le travail au cours des dix ans de recherche sur le système Lumidyne donne comme résultat : « more than 100 kinetic paintings [...] rang[ing] in size from $25 \mathrm{~cm} \mathrm{x} 25 \mathrm{~cm}$ to $3 \times 3.5$ $\mathrm{m} »$ (MALINA, 1968, p. 33).

Extrêmement rigoureux dans la structure autant que dans l'argumentation, ce texte se rapproche de la facture académique. Cela n'empêche pas Malina d'avancer des arguments non fondés sur des faits, notamment dans la partie des notions. Tel que Noël, il avance des idées, parfois, complexes de placer d'un système vérité scientifique. Bien que les 
écrits chez Leonardo suivent les critères des publications académiques, ils échappent pourtant à l'objectivité attendue par ceux-ci. On se demande alors pourquoi.

Finalement, une troisième forme de critique développée par les artistes, est celle où il n'y a pas d'évocations de quelconque fait personnel, juste le développement des notions. C'est des écrits à caractère théorique.

C'est le cas des écrits de Philip Pavia dans la revue It is. A Magazine for Abstract Art. Permettant de comprendre le moment particulier traversé par le milieu artistique nord-américain à la fin des années cinquante, cette revue témoigne du scénario d'avant-garde américain après 1945. Rédigeant l'article éditorial de chaque numéro, intitulé tous manifesto-in-progress, Pavia constitue au fil de publications, une réflexion théorique sur l'expressionisme abstrait.

La particularité de ses écrits se trouve dans le fait que, suivant les formalités de la critique artistique et de la théorique, il frôlent cependant avec un travail littéraire : "Non-history is a sense of art with the object left out » (PAVIA, 1958-1959, p. 8). Bien que l'on peut arriver à comprendre le sens de ce qui est affirmé, de la même manière qu'avec les autres artistes, on observe que les écrits comportent des phrases que vont au-delà de la rigueur académique. Même quand le but est d'expliquer et clarifier des éléments, certains écrits restent à mi chemin entre celle-ci et la création littéraire.

\section{Nouvelle catégorie discursive : la critique d'art comme pratique poético-artistique}

Ces textes n'auront pas seulement contribué à une diversification des tribunes à partir desquelles les artistes s'adressaient au monde, mais aussi à la transmission et à la circulation de la parole, suivant une échelle encore inétendue au début des années cinquante. Attachés directement ou indirectement à l'objet artistique, étant ou non de l'art, ils jouent un rôle dans la définition des nouveaux rapports institutionnels, plus précisément, en mettant en place des nouvelles formes d'expression. Comme avec une carte géographique, cela implique un déplacement des anciennes frontières discursives.

En effet, l'écriture des artistes mène vers un nouveau type discursif. Les trois degrés d'implication des artistes que l'on a pu étudier, montrent bien que, si bien le degré de subjectivité varie selon les écrits, une même 
caractéristique persiste : une manière d'expression littéraire voire artistique qui se mêle de l'ensemble des thèses avancées. Si bien que les artistes cherchant à apporter un point de vue critique qui puisse être validé par le monde de l'art, cela a eu lieu en ayant recours à leurs propres impressions sur ce qui doit être la création artistique, depuis leur rôle en tant qu'artistes. Cela comporte deux conséquences essentielles.

D'abord, tout degré d'objectivité est forcément biaisé par le fait que l'artiste parle de son œuvre. La valeur des textes ne peut pas donc être cherchée du côté de leur impartialité. Volontairement ou involontaire, les artistes cherchent à promouvoir leurs propres pratiques. Dans ce sens, ces textes doivent être compris, bien que l'argumentaire comporte toute la valeur d'un texte critique réalisé par un non-artiste avec le niveau d'objectivité que cela peut impliquer, comme une ouverture au monde intellectuel des artistes mêmes. Leurs textes sont donc un prolongement de leurs vies. Ce sont des dialogues ouverts établis avec une troisième personne, dans ce cas là, le spectateur, potentiel lecteur.

Ensuite, puisque l'on ne cherche pas une vérité qui puisse se dévoiler au fil de l'argumentation, mais plutôt à rentrer dans l'univers intellectuel des artistes, il est compréhensible, que cela se fasse à partir des limites conceptuelles établies par les artistes. En effet, saisir leur pensée suppose, pouvoir se laisser emporter par la dimension esthétique des affirmations.

La lecture de ces écrits suppose alors de se positionner face à une structure argumentative qui alterne à la fois un rapport avec contemplation esthétique des mots qui ne cherchent pas à véhiculer une vérité sur le monde, mais plutôt une vision du monde.

\section{Références}

ALLEN, Gwen. Artists' Magazines: an Alternative space for art. Cambridge (MA): The MIT Press, 2011.

COMETTI, Jean-Pierre ; MORIZOT, Jacques ; POUIVET, Roger. Questions d'esthétique. Paris : Presses Universitaires de France, 2000.

CORBEL, Laurence. Le Discours de l'art : écrits d'artistes 1960-1980. Rennes: Presses Universitaires de Rennes, 2012.

FLAVIN, Dan. ...«In daylight or cool white.» An Autobiographical Sketch. Artforum, v. 4, Décembre, 1965, p. 22-25. 
HIGGINS, Dick. Postface - Un journal critique de l'avant-garde (1964). Dijon : Les Presses du réel, 2005.

KUSAMA, Yayoi. Manhattan suicide addict (1978). Traduction du japonais par Isabelle Charrier, Dijon : Les Presses du réel, 2005.

MALINA, Frank J. Kinetic painting: The Lumidyne system. Leonardo, n. 1, 1968, p. 25-33.

MIGNON, Oliver (éd.). L'artiste comme théoricien. Réciprocité entre théorie et pratique artistique de 1965 à 1985, France : Les Presses du réel, 2010.

MCEGLIN-DELCROIX, Anne. Esthétique du livre d'artiste 1960-1980. Paris : Bibliothèque National de France / Jean-Michel Place, 1997.

NOËL, Georges. Reliefs. Leonardo, n. 1, 1968, p. 77-80.

OLDENBURG, Claes. Extracts from the Studio Notes. Artforum, v. 4, Janvier, 1966, p. 33-36.

PAVIA, Philip. Manifesto -in- progress. It is. A Magazine for Abstract Art, n. 1-5, 1958-1959.

SECUNDA, Arthur. Tinguely and Rickey, two motion sculptors. Artforum, v. 1, Juin, 1962, p. 17-19.

. John Bernhardt, Charles Frazier, Edward Kienholz. Artforum, v. 1, Octobre, 1962, p. 30-34.

. Rico Lebrun, An interview. Artforum, v. 1, Mai, 1963, p. 34-36. 